\title{
Dynamic Behavior of Vortices under Applied Current Drive in Mesoscopic Superconducting Strip with Two Magnetic Dots
}

\author{
Lin PenG ${ }^{a, *}$, Yajing $\mathrm{WANG}^{a}$, Yufeng Zhang ${ }^{a}$, JiAnming Xu ${ }^{b}$ And Lina SAnG ${ }^{c}$ \\ ${ }^{a}$ Department of Physics, Shanghai University of Electric Power, Shanghai 201300, China \\ ${ }^{b}$ Shanghai Institute of Space Power-Sources, Shanghai 200245, China \\ ${ }^{c}$ Institute for Superconducting and Electronic Materials, Faculty of Engineering, \\ Australian Institute for Innovative Materials, University of Wollongong, NSW 2500, Australia
}

(Received September 24, 2019; revised version November 9, 2019; in final form November 19, 2019)

Using a finite element method to numerically solve the time-dependent Ginzburg-Landau equations, we study the dynamic behavior of vortices in a hybrid structure consisting of a superconducting strip with two magnetic dipoles in the presence of a external homogeneous magnetic field and an applied current drive. Time-averaged voltage-current, free energy, density of Cooper pairs, and vortex dynamics in the superconducting strip were obtained. From the obtained results, the period of free energy oscillation is observed. The period of free energy oscillation corresponds to the period of vortices variation under the applied currents drive. The obtained results give a better understanding of free energy oscillation and vortices variation in the mesoscopic superconducting strip under an applied current drive.

DOI: 10.12693/APhysPolA.137.385

PACS/topics: free energy oscillation, dynamic behavior of vortices, magnetic dipoles, the time-dependent GinzburgLandau equations, finite element method.

\section{Introduction}

Superconductivity at reduced structure dimensions has received an increasing interest mainly due to the huge advancements in modern nanofabrication techniques, which have permitted exploration and discovery of new physical phenomena when approaching the mesoscopic limit (dimensions of the order of coherence length $\xi$ or penetration depth $\lambda$ ), such as the giant vortex state [1], multivortex state [2], vortex-antivortex [3], paramagnetic Meissner effect [4], symmetry-induced antivortices [5], fractional flux vortices [6], and vortices trapped in blind holes [7]. The physics becomes even richer when an electric field is applied to the system in addition to a magnetic field, which will result in the phase-slip phenomenon [8], magnetoresistance oscillations [9], fast moving kinematic vortices [10], and stroboscopic resonances phenomena [11].

The mesoscopic superconductors have been considered extensively both experimentally and theoretically [1-11] up to now. For theoretic studies, the Ginzburg-Landau (GL) theory [12] is probably the most successful macroscopic description of superconductivity [13]. Technically speaking, GL theory is only valid close to the normalsuperconductor phase boundary where the order parameter is small. However, empirically it appears to have a rather broader range of applications than this, and even deep in the superconducting state (where magnetic imaging is performed), we find excellent qualitative

*corresponding author; e-mail: plpeng@shiep.edu.cn agreement with GL predictions [13]. In this paper, we investigated a mesoscopic two-dimensional superconducting strip with two magnetic dots under an applied current drive, and time-averaged voltage, free energy, density of the Cooper pairs, and vortex dynamics in the superconducting strip were obtained. Numerical simulations will be performed in the framework of the time-dependent Ginzburg-Landau (TDGL) model. The finite-element method (FEM) [14-16] was used due to its ability to handle complex geometries and complicated boundary conditions, and the additional benefit of less computing time. The TDGL equations are numerically solved to obtain the dynamic properties of the superconducting strips. The work is organized as follows. In Sect. 2, we show the derived TDGL equations and explain the numerical method and procedure used in the calculations. In Sect. 3, we analyze the results obtained for the superconducting strips. Our results are finally summarized in Sect. 4.

\section{Time-dependent Ginzburg-Landau method}

We consider a mesoscopic rectangle-shaped superconducting strip (see Fig. 1). Two point-like out-of-plane magnetic dipoles are separated by a difference $d$ between their $x$-coordinates and placed at one distance $Z_{1}$ and another distance $Z_{2}$ from the top surface of the superconducting strip. In our model, the effect of two magnetic dipoles is equivalent to a magnetic strip. In other words, the magnetic strip can create a stray magnetic which in turn can be modeled by two point-like magnetic dipoles directed perpendicular to the superconductor [17]. We do not change the size of the superconducting strip for all considered four cases. The strip is surrounded by 


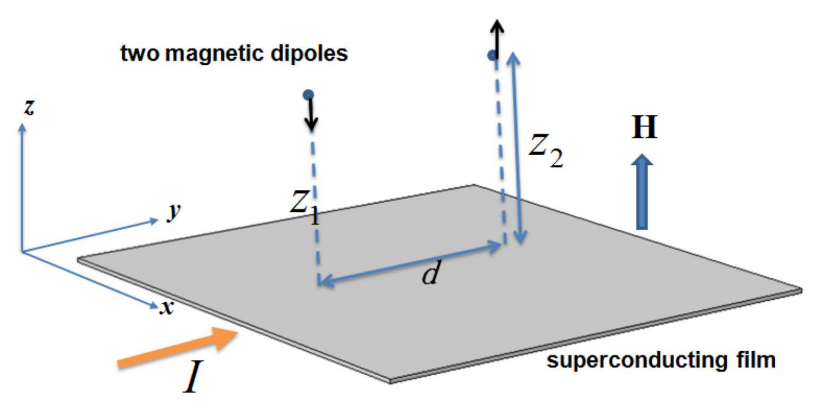

Fig. 1. A sketch of the envisioned experimental setup. Two point-like out-of-plane magnetic dipoles are separated by a difference $d$ between their $x$-coordinates and placed at one distance $Z_{1}$ and another distance $Z_{2}$ from the top surface of the superconducting strip with dimensions $L_{x} \times L_{y}=6 \xi \times 15 \xi$. The superconducting strip is under applied dc current $\boldsymbol{I}$ and a perpendicular magnetic field $\boldsymbol{H}$. A magnetic dipole has magnetic moment $\boldsymbol{m}=\left(0,0, m_{z}\right)$.

vacuum with an applied magnetic field $\boldsymbol{H}=(0,0, H)$ in the $z$-direction and the transport current $\boldsymbol{I}=(0, I, 0)$ in the $y$-direction. The GL theory describes the superconducting state through a complex order parameter $\psi$ for which $|\psi|^{2}$ represents the density of the Cooper pairs. The order parameter and the free energy can be determined by the TDGL equations, which are expressed by

$$
\begin{aligned}
& \left(\frac{\partial}{\partial t}+\mathrm{i} \Phi\right) \psi=-(-\mathrm{i} \nabla-\boldsymbol{A})^{2} \psi+\left(1-|\psi|^{2}\right) \psi \\
& \sigma\left(\frac{\partial \boldsymbol{A}}{\partial t}+\nabla \Phi\right)=\boldsymbol{J}_{s}-\kappa^{2} \nabla \times \nabla \times \boldsymbol{A} \\
& \boldsymbol{J}_{s}=\operatorname{Im}\left(\psi^{*} \nabla \psi\right)-\boldsymbol{A}|\psi|^{2}
\end{aligned}
$$

where $\Phi$ is the electric potential, $\boldsymbol{A}$ is the vector potential, $\sigma$ is the normalized conductivity, $\boldsymbol{J}_{s}$ is the superconducting current density. The vector potential $\boldsymbol{A}$ can be represented as $\boldsymbol{A}=\boldsymbol{A}_{m}+\boldsymbol{A}_{1}+\boldsymbol{A}_{0}$, where $\boldsymbol{A}_{m}$ corresponds to the magnetic field, induced by the two magnetic dipoles (the magnetic moment $\boldsymbol{m}=\left(0,0, m_{z}\right)$ ), and $\boldsymbol{A}_{1}$ denotes the vector potential corresponding to an additional external homogeneous magnetic field $\boldsymbol{H}$. The vector potential $\boldsymbol{A}_{0}$ describes magnetic field induced by the currents, which flow in the superconducting strip. We may neglect both variations in the order-parameter magnitude and the $\boldsymbol{A}_{0}$ contribution because the thickness of the superconducting strip is assumed to be sufficiently small as compared to $\xi$ and $\lambda$.

We scale the length in units of $\xi=\hbar / \sqrt{2 m\left|\alpha_{0}\right|}$, the order parameter $\psi$ in units of $\psi_{0}=\sqrt{-\alpha_{0} / \beta}$ (with $\alpha_{0}$ and $\beta$ being the GL coefficients [18]), $\boldsymbol{A}$ is given in units of $\Phi_{0} /(2 \pi \xi)\left(\Phi_{0}=c h /(2 e)\right.$ is the flux quantum). The magnetic field is in units of $H_{c 2}=\Phi_{0} /\left(2 \pi \xi^{2}=\sqrt{2} \kappa H_{c}\right)$, where $H_{c}$ is the thermodynamic critical field, $\kappa=\lambda / \xi$ is the GL parameter. The time is in units of GL relaxation time $t_{0}=\pi \hbar /\left(8 k_{\mathrm{B}} T_{c}\right)$. We scale the $m$ in units of $m_{0}=\Phi_{0} \xi /(2 \pi)$. The applied current density is given in units of $j_{0}=\sigma_{n} \hbar /\left(2 e t_{0} \xi\right)$ ( $\sigma_{n}$ is the normal-state conductivity), and the voltage scale is given by $V_{0}=\hbar /\left(2 e t_{0}\right)$. The free energy of the superconducting state, measured in $F_{0}=H_{c}^{2} V /(8 \pi)$ units, is expressed as

$$
\begin{aligned}
F & =\frac{2}{V} \int\left[-|\psi|^{2}+\frac{1}{2}|\psi|^{4}+|(-\mathrm{i} \nabla-\boldsymbol{A}) \psi|^{2}\right. \\
& \left.+\kappa^{2}(\boldsymbol{B}-\boldsymbol{H})^{2}\right] \mathrm{d} V .
\end{aligned}
$$

For the magnetic field, the boundary condition reads: $\left.(\boldsymbol{\nabla} \times \boldsymbol{A})\right|_{\text {boundary }}=\boldsymbol{H}$. The transport current is introduced via the boundary condition for the vector potential in the $x$ direction: $\boldsymbol{\nabla} \times\left.\boldsymbol{A}\right|_{z}(x=0, w)=H \pm H_{I}$, where $H_{I}=2 \pi I / c$ is the magnetic field by the current $I$. From the vector potential it is possible to obtain the voltage by using the $V=\frac{\partial}{\partial t} \int \boldsymbol{A} \mathrm{d} \boldsymbol{l}$. For the order parameter, we use the superconductor-insulator boundary conditions, i.e., we set the normal component of the supercurrent across the boundary to zero: $\left.\boldsymbol{n} \cdot(-\mathrm{i} \boldsymbol{\nabla}-\boldsymbol{A}) \psi\right|_{\text {boundary }}=0$, where $\boldsymbol{n}$ is the outward normal unit to the surface. Finally, to simulate a infinite length strip, we apply periodic boundary conditions in the $y$-direction. The TDGL equations and their discrete form are gauge invariant under the transformations as follows: $\psi^{\prime}=\psi \mathrm{e}^{\mathrm{i} \chi}, \boldsymbol{A}^{\prime}=\boldsymbol{A}+\boldsymbol{\nabla} \chi$, $\Phi^{\prime}=\Phi-\partial \chi /(\partial t)$. We chose the zero-scalar potential gauge, that is, $\Phi=0$ at all times and positions.

\section{Results and discussions}

We first consider the mesoscopic superconducting strip with sizes of $L_{x} \times L_{y}=6 \xi \times 15 \xi$. Our simulations have been carried out by using $\sigma=1, \kappa=1.2$, and $d=6 \xi$ for the superconducting strip. The initial conditions are $|\psi|^{2}=1$ corresponding to the Meissner state and zero magnetic field inside the superconductor. One magnetic dipole $m_{1}$ is located at site $\left(3,4.5, Z_{1}\right)$, and another $m_{2}$ is located at site $\left(3,10.5, Z_{2}\right)$. We restrict ourselves to the case when the stray fields of the two magnetic dots are weak, so that no vortex-antivortex pairs are created at zero applied current. Figure 2a shows time-averaged voltage as a function of applied current $(V(I))$ at $H / H_{c 2}=1.2, m_{1}=-20, \quad m_{2}=20$, and $Z_{1}=Z_{2}=6$.
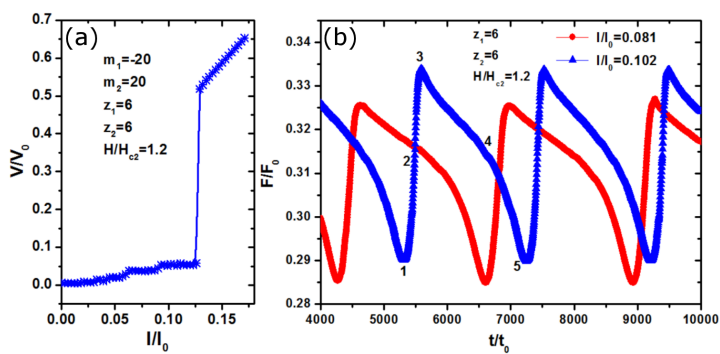

Fig. 2. (a) Time-averaged voltage as a function of the applied current in the superconducting strip. (b) Free energy vs. time characteristics of the strip at $Z_{1}=Z_{2}=6$ and $H / H_{c}=1.2$. 

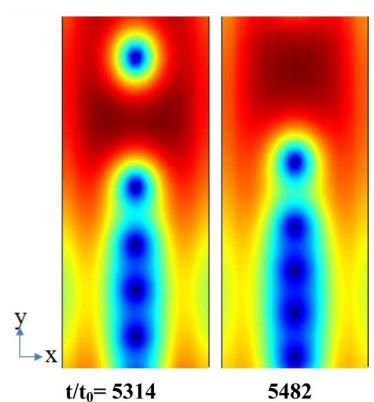

5482

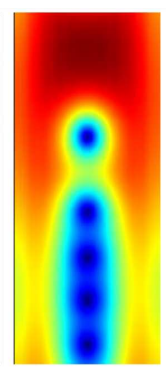

5587

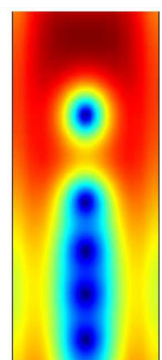

6505

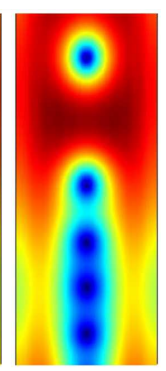

7219

Fig. 3. Contour plots of the Cooper-pair density at $I / I_{0}=0.102$. Blue to red means that the absolute value of the order parameter ranges from minimum to maximum.
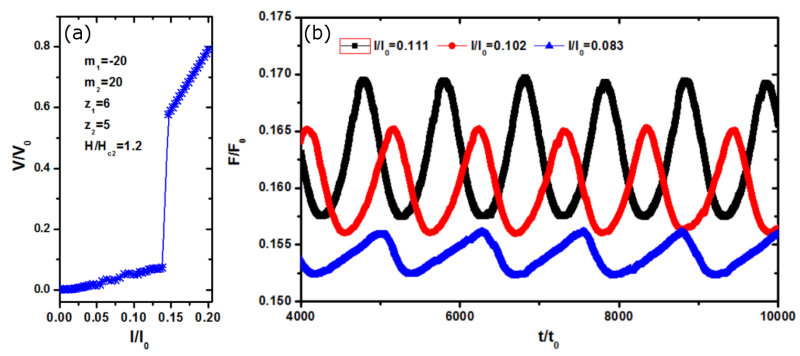

Fig. 4. (a) Time-averaged voltage as a function of the applied current in the superconducting strip. (b) Free energy vs. time characteristics of the strip at $Z_{1}=6$, $Z_{2}=5$ and $H / H_{c 2}=1.2$.

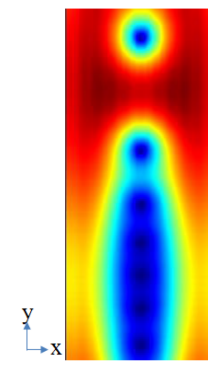

$t / t_{0}=6282$

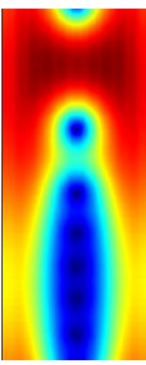

6588

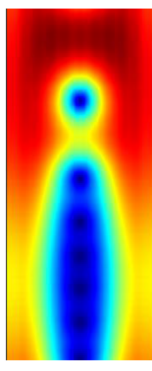

6865

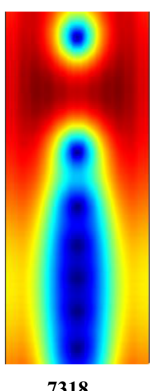

7318

Fig. 5. Contour plots of the Cooper-pair density at $I / I_{0}=0.102$. Blue to red means that the absolute value of the order parameter ranges from minimum to maximum.

It is known that the superconducting strip goes through two levels of increasing resistance as driving current is increased. Low resistance of the superconducting strip is maintained up to a threshold current when maximal current in the strip reaches the de-pairing current. The threshold current is $I_{c} / I_{0}=0.125$. With further increase in applied current, superconductivity becomes utterly suppressed in the strip, leading to a normal state of higher resistance. Figure $2 \mathrm{~b}$ shows free energy as a function of the time $(F(t))$ at $H / H_{c}=1.2, Z_{1}=Z_{2}=6$
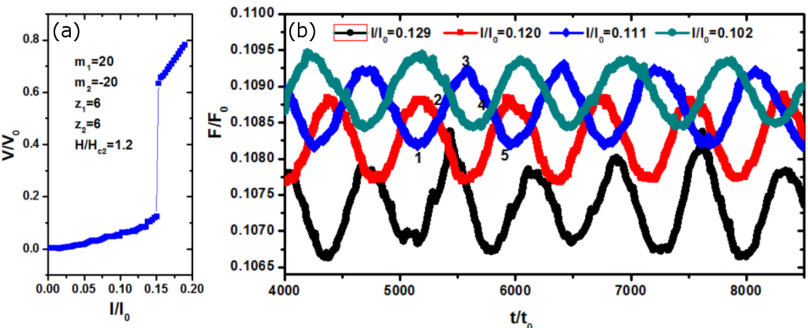

Fig. 6. (a) Time-averaged voltage as a function of the applied current in the superconducting strip. (b) Free energy vs. time characteristics of the strip at $Z_{1}=Z_{2}=$ 6 and $H / H_{c}=1.2$.
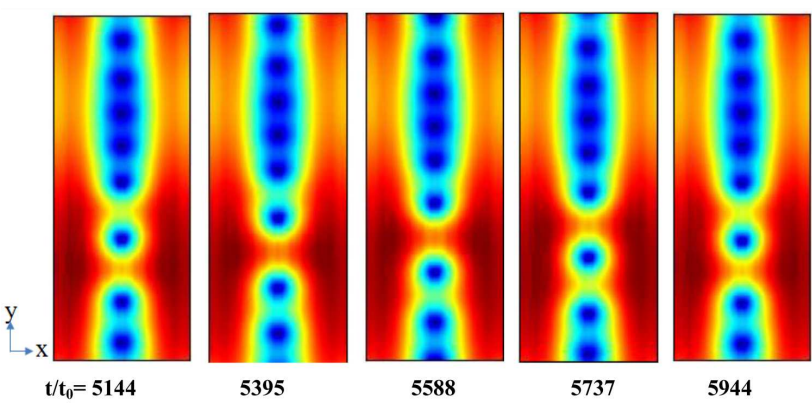

Fig. 7. Contour plots of the Cooper-pair density at $I / I_{0}=0.120$. Blue to red means that the absolute value of the order parameter ranges from minimum to maximum.

for $I / I_{0}=0.102$ and $I / I_{0}=0.081$, respectively. For the chosen length of simulation region and the considered magnetic field, we actually had $N_{v}=5$ vortices moving in a single row, as shown in the contour plots of the Cooper-pair density in Fig. 3. The interplay of the vortices and currents leads to the free energy oscillation. Namely, the applied magnetic field and the magnetic field of magnetic dots induce reactive screening (and circulating) currents in the superconductor, $I_{s}$. The applied dc current always enhances the supercurrent $I_{s}$ on one side of the strip, while suppressing $I_{s}$ on the other side. This directly affects the barriers for vortex entry and exit, which is indicated in Fig. $2 \mathrm{~b}$ and 3 . There, points $1-5$ are used to denote one period of the vortex dynamics, making the characteristic time intervals. We could observe the period is $\tau / t_{0} \sim 2000$.

Figure 4a shows time-averaged voltage as a function of applied current $(V(I))$ at $m_{1}=-20, m_{2}=20, Z_{1}=6$, $Z_{2}=5$, and $H / H_{c 2}=1.2$, which shows that the threshold current is $I_{c} / I_{0}=0.138$. Figure $4 \mathrm{~b}$ shows free energy as a function of the time $(F(t))$ at $Z_{1}=6, Z_{2}=5$, and $H / H_{c 2}=1.2$ for $I / I_{0}=0.083, I / I_{0}=0.102$ and $I / I_{0}=0.111$, respectively. We could observe that the oscillation period of free energy is $\tau / t_{0} \sim 1000$. For the chosen conditions of simulation we actually had $N_{v}=7$ vortices moving in a single row, as shown in the contour plots of the Cooper-pair density in Fig. 5. 


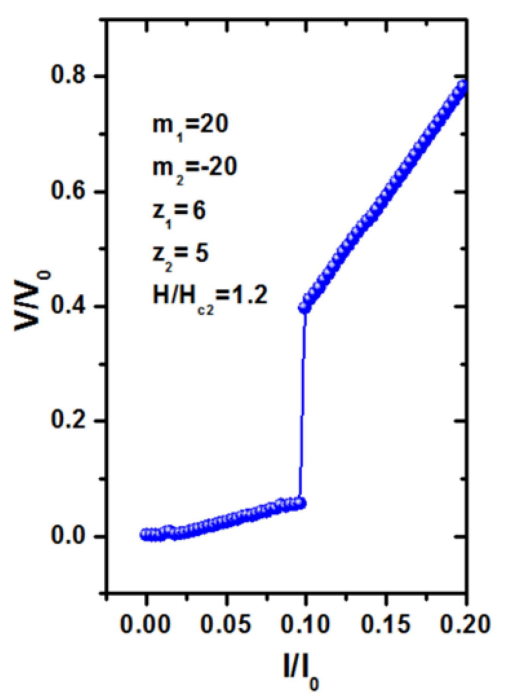

Fig. 8. Time-averaged voltage as a function of the applied current in the superconducting strip.

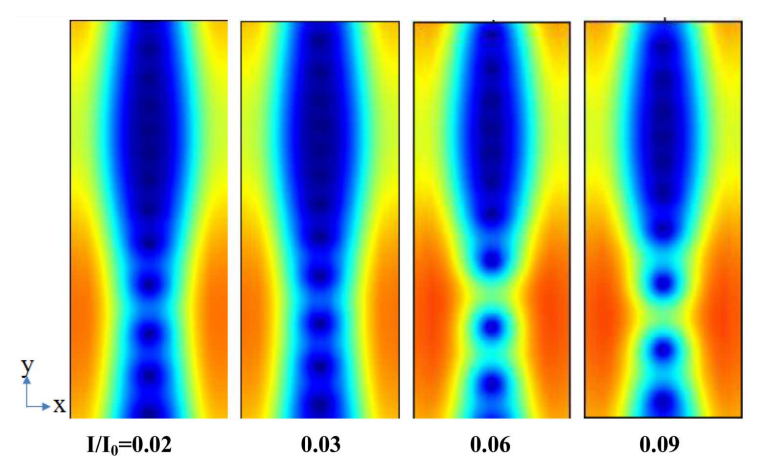

Fig. 9. Contour plots of the Cooper-pair density at the $I / I_{0}=0.02,0.03,0.06$, and 0.09 . Blue to red means that the absolute value of the order parameter ranges from minimum to maximum.

Up to now we studied the dynamic properties of the superconducting strip when $m_{1}=-20, m_{2}=20$. In what follows, we consider the case for $m_{1}=20, m_{2}=-20$. Figure 6 a shows the time-averaged voltage as a function of the applied current in the superconducting strip at $H / H_{c 2}=1.2, m_{1}=20, m_{2}=-20$, and $Z_{1}=Z_{2}=6$. We observed that the threshold current was $I_{c} / I_{0}=0.15$. Figure $6 \mathrm{~b}$ shows free energy as a function of the time $(F(t))$ at $H / H_{c}=1.2, Z_{1}=Z_{2}=6$ for $I / I_{0}=0.081$, $I / I_{0}=0.111, I / I_{0}=0.120$, and $I / I_{0}=0.129$, respectively. For the chosen conditions of simulation we actually had $N_{v}=8$ vortices moving in a single row, as shown in the contour plots of the Cooper-pair density in Fig. 7. There, points $1-5$ are used to denote one period of the vortex dynamics, making the characteristic time intervals. We could observe that the oscillation period of free energy is $\tau / t_{0} \sim 800$. Figure 8 shows the time-averaged voltage as a function of the applied current in the superconducting strip at $m_{1}=20, m_{2}=-20$, $Z_{1}=6, Z_{2}=5$, and $H / H_{c 2}=1.2$. We observed that the threshold current was $I_{c} / I_{0}=0.10$. Figure 9 shows the contour plots of the Cooper-pair density at the $I / I_{0}=0.02,0.03,0.06$, and 0.09 . Under the drive of different applied currents, we observed the different distribution characteristics of vortices. With increasing current, the motion of the vortices speed up.

\section{Conclusions}

Using the TDGL theory we studied the dynamic properties of the superconducting strips with two magnetic dipoles in the presence of a external homogeneous magnetic field and an applied current drive. Through our research, the dynamic properties of a superconducting strip were obtained for two different directions of magnetic moments of the magnetic dots. We observed that the period of free energy oscillation at the case of $m_{1}=-20$ and $m_{2}=20$ was larger than the one in the case of $m_{1}=20$, and $m_{2}=-20$. The period of free energy oscillation corresponds to the period of vortices variation, which was induced by the applied currents. The obtained results would be helpful to understand the characteristics of vortices of mesoscopic superconductors in experiments.

\section{Acknowledgments}

This work is sponsored by the Natural Science Foundation of Shanghai (No. 17ZR1411400, 16ZR1422700), the Opening Project of Shanghai Key Laboratory of High Temperature Superconductors (No. 14DZ2260700).

\section{References}

[1] T. Cren, L. Serrier-Garcia, F. Debontridder, D. Roditchev, Phys. Rev. Lett. 107, 097202 (2011).

[2] G.R. Berdiyorov, M.V. Milošević, S. Savel'ev, F. Kusmartsev, F.M. Peeters, Phys. Rev. B 90, 134505 (2014).

[3] M.V. Milošević, W. Gillijns, A.V. Silhanek, A. Libál, V.V. Moshchalkov, F.M. Peeters, Appl. Phys. Lett. 96, 032503 (2010).

[4] A.K. Geim, S.V. Dubonos, J.G.S. Lok, M. Henini, J.C. Maan, Nature 396, 144 (1998).

[5] L.F. Chibotaru, A. Ceulemans, V. Bruyndoncx, V.V. Moshchalkov, Nature 408, 833 (2000).

[6] A.K. Geim, S.V. Dubonos, I.V. Grigorieva, K.S. Novoselov, F.M. Peeters, V.A. Schweigert, Nature 407, 55 (2000).

[7] A. Bezryadin, Yu.N. Ovchinnikov, B. Pannetier, Phys. Rev. B 53, 8553 (1996).

[8] G.R. Berdiyorov, M.V. Milošević, F.M. Peeters, Phys. Rev. B 80, 214509 (2009).

[9] G.R. Berdiyorov, M.V. Milošević, M.L. Latimer, Z.L. Xiao, W.K. Kwok, F.M. Peeters, Phys. Rev. Lett. 109, 057004 (2012). 
[10] L.N. Bulaevskii, Matthias J. Graf, V.G. Kogan, Phys. Rev. B 85, 014505 (2012).

[11] Ž. L. Jelić, M.V. Milošević, A.V. Silhanek, Sci. Rep. 6, 35687 (2016).

[12] V.L. Ginzburg, L.D. Landau, "On the Theory of Superconductivity" in: On Superconductivity and Superfluidity, Springer, Berlin 2009, p. 113.

[13] S.J. Bending, M.V. Milošević, V.V. Moshchalkov, in: Nanoscience and Engineering in Superconductivity, Eds. V. Moshchalkov, R. Wöerdenweber, W. Lang, Springer, Berlin 2009, p. 299.
[14] H.D. Gao, W.W. Sun, J. Comput. Phys. 294, 329 (2015).

[15] Q. Du, Phys. Rev. B 46, 9027 (1992).

[16] L. Peng, Y. Hu, Z. Li, K. Deng, Y. Zhu, L. Xu, Y. Zhou, Acta Phys. Pol. A 133, 152 (2018).

[17] A.V. Kapra, V.R. Misko, D.Y. Vodolazov, F.M. Peeters, Supercond. Sci. Technol. 24, 024014 (2011).

[18] C.Y. Liu, G.R. Berdiyorov, M.V. Milošević, Phys. Rev. B 83, 104524 (2011). 